\title{
Consumo de lipídeos e estado nutricional de idosos participantes do projeto NUTENV da Universidade de Caxias do Sul
}

\author{
Marilza de Oliveira Rezer Kucera*, Josiane Siviero*, Simone Bonatto***
}

\section{Resumo}

O objetivo deste estudo foi avaliar, por meio da antropometria, o consumo alimentar de lipídeos, ácidos graxos monoinsaturados, poli-insaturados, saturados e colesterol, bem como o estado nutricional de idosos participantes do projeto NUTENV da Universidade de Caxias do Sul - RS. Trata-se de um estudo transversal, retrospectivo, descritivo e analítico, com 90 idosos de ambos os sexos. Foram analisadas variáveis demográficas, socioeconômicas e de consumo alimentar. Em relação às variáveis antropométricas, foram avaliados o IMC e CC. Para estimar o consumo alimentar, utilizou-se o R24h. A análise estatística envolveu o teste qui-quadrado de Pearson para associação dos marcadores antropométricos (IMC e CC) com as variáveis demográficas e socioeconômicas e o coeficiente de correlação de Pearson para correlacionar o consumo de lipídeos com os marcadores antropométricos e a variável demográfica idade. O consumo de lipídeos foi descrito através de média, mediana, mínimo, máximo e desvio padrão. Entre os indivíduos analisados, houve prevalência do sexo feminino, casados, com renda mensal entre 2 e 4 salários-mínimos, e mais de 8 anos de escolaridade. Na avalia- ção antropométrica, 71,1\% dos indivíduos estão com risco elevado ou muito elevado para DCV, considerando a CC. Por meio do IMC, obteve-se uma maior frequência de obesidade $(56,7 \%)$. O consumo dos lipídeos totais ficou abaixo do recomendado para a maioria dos indivíduos analisados. Para os ácidos graxos e colesterol, obteve-se resultados de consumo dentro do recomendado. $\mathrm{Na}$ associação das variáveis, os resultados demonstraram uma maior prevalência de obesidade entre os idosos com 60-69 anos e viúvos. Houve significância negativa entre o consumo de lipídeos e o IMC, registrando-se que quanto maior o consumo de lipídeos menor o IMC. Torna-se necessária a adoção de hábitos alimentares saudáveis, com uma dieta balanceada de macro e micronutrientes, associada à prática de atividade física, como uma das estratégias de diminuição de CC e IMC. Sugere-se, ainda, que novos estudos sejam realizados relacionando o consumo de lipídeos em indivíduos em processo de envelhecimento, utilizando outros instrumentos de avaliação.

Palavras-chave: Ácidos graxos. Envelhecimento. Estado nutricional. Idoso.

* Acadêmica do curso de graduação em Nutrição da Universidade de Caxias do Sul. Endereço para correspondência: Rua Major Adauto Cruz, 1449, apto. 302, Bairro Sagrada Família, CEP: 95054-270, Caxias do Sul - RS. E-mail: marirezer@yahoo.com.br.

** Nutricionista. Doutora em Ciências da Saúde pela Pontifícia Universidade Católica do Rio Grande do Sul (PUCRS). Mestra em Gerontologia Biomédica pelo Instituto de Geriatria e Gerontologia (IGG/PUCRS). Docente do curso de Nutrição da Universidade de Caxias do Sul e da Faculdade Cenecista de Bento Gonçalves (CNEC).

*** Nutricionista, Mestre em Saúde Coletiva. Docente do Curso de Nutrição da Universidade de Caxias do Sul.

$\hookrightarrow$ doi:10.5335/rbceh.2012.041 


\section{Introdução}

O envelhecimento da população mundial vem sendo bastante discutido na última década, por se tratar de um fenômeno universal. No Brasil, tem ocorrido com rapidez, já havendo cerca de $10 \%$ de idosos (ROQUE; BOMFIM; CHIARI, 2010). No processo de envelhecimento, a importância de uma alimentação adequada é comprovada por estudos epidemiológicos, clínicos e de intervenção, entre outros, que têm demonstrado ligação consistente entre $o$ tipo de dieta e o surgimento de doenças crônicas não transmissíveis, incluindo as doenças cardiovasculares (DCV). (CERVATO et al., 2005).

Dentre as doenças, as DCV são as principais causas de morte entre adultos no mundo todo. (DURO; ASSUNÇÃO; COSTA, 2008). São considerados fatores de risco para a prevalência das dislipidemias as características étnicas, socioeconômicas e culturais de grupos populacionais distintos, coexistindo com fatores como sobrepeso, obesidade, hipertensão arterial, dislipidemias e diabetes. (BORTOLI et al., 2011). Em relação aos nutrientes, destaca-se a atenção ao consumo de lipídeos saturados e insaturados em idosos.

Os ácidos graxos poli-insaturados são encontrados em uma variedade de alimentos de origem vegetal, que constituem dietas comprovadamente redutoras do risco de DCV. Trata-se de lipídeos essenciais ao desenvolvimento e à manutenção do organismo. (SILVA; SÁ, 2012). As altas ingestões de gorduras saturadas estão relacionadas a resis- tência insulínica e hiperinsulinemia, aumentando o risco de desenvolvimento dessa doença. Vários estudos prospectivos têm associado a ingestão dietética de gorduras ao risco de desenvolver Doença de Alzheimer. (MACHADO; FRANK; SOARES, 2006). A gordura monoinsaturada tem a capacidade de remover radicais livres e reduzir a síntese de citocinas pró-inflamatórias no tecido adiposo e, assim, reduzir o estímulo à síntese hepática de proteínas de fase aguda. Evidências científicas acumuladas sugerem a redução dos fatores de risco tradicionais que definem a síndrome metabólica e as doenças cardiovasculares. (JENKINS et al., 2010; LATREILLE et al., 2012).

Sendo assim, o objetivo deste estudo foi determinar o consumo de lipídeos (lipídeos totais, ácidos graxos saturados, monoinsaturados, poli-insaturados e colesterol) e o estado nutricional e antropométrico em idosos participantes do projeto Nutrição e Envelhecimento (NUTENV) da Universidade de Caxias do Sul (UCS).

\section{Metodologia}

Trata-se de um estudo do tipo transversal, retrospectivo, descritivo e analítico. A amostra foi constituída por 90 idosos participantes do projeto NUTENV da UCS, intitulado "Alimentação e envelhecimento: estudo do perfil nutricional, antropométrico e a relação com fatores socioeconômicos e culturais de idosos participantes da Universidade da Terceira Idade (UNTI)", aprovado pelo Comitê de Ética em Pesquisa da UCS, sob o número 061/2009. 
Participaram deste estudo os indivíduos de ambos os sexos que atenderam aos seguintes critérios de inclusão: ter idade $\geq 60$ anos, ser participante da UNTI da UCS e assinar o Termo de Consentimento Livre e Esclarecido. A coleta de dados ocorreu no Laboratório de Avaliação e Educação Nutricional da UCS. Os participantes da UNTI eram convidados a participar do projeto; havendo interesse, era agendada uma entrevista com data e horário pré-determinados. Os participantes eram pesados e medidos, tinham a sua circunferência de cintura (CC) aferida e respondiam a um questionário demográfico e socioeconômico, além de um recordatório alimentar $24 \mathrm{~h}$ (R24h). (KARVETTI; KNUTS, 1985). Todos esses processos metodológicos eram desenvolvidos por acadêmicas de nutrição previamente treinadas.

Os dados foram coletados de 2010 a 2012. Foram utilizadas, neste estudo, como variáveis demográficas: idade (6069 anos e $\geq 70$ anos), gênero (feminino ou masculino) e estado civil (solteiro/casado/ divorciado ou viúvo). Como variáveis socioeconômicas, considerou-se: renda (em salários-mínimos) e escolaridade (categorizada em ensino fundamental, ensino médio e superior). As variáveis antropométricas foram definidas por: índice de massa corporal (IMC) e CC. Por fim, foram estabelecidos como variáveis alimentares: lipídeos totais, colesterol, ácidos graxos saturados, monoinsaturados e poli-insaturados.

O IMC foi obtido pelo resultado da divisão do peso corporal em $\mathrm{kg}$ pelo quadrado da estatura em metros. O peso corporal foi aferido por balança mecânica da marca Cauduro ${ }^{\circledast}$. A estatura foi obtida através do estadiômetro acoplado à balança. Os indivíduos foram pesados e medidos em posição ortostática, com a cabeça para frente, os pés juntos, sem sapatos e com o mínimo de roupa possível. O estado nutricional, segundo o IMC, foi analisado considerando as referências de Lipschitz (1994), sendo: eutrofia $\left(22-27 \mathrm{~kg} / \mathrm{m}^{2}\right)$; desnutrição $\left(<22 \mathrm{~kg} / \mathrm{m}^{2}\right)$ e obesidade $\left(>27 \mathrm{~kg} / \mathrm{m}^{2}\right)$. Em relação à CC, o critério utilizado para classificar o risco nutricional entre os homens foi 94-101 cm como sendo risco aumentado para DCV e $\geq 102 \mathrm{~cm}$ como risco muito aumentado; para as mulheres, foi considerado 80-87 cm como risco aumentado e $\geq 88 \mathrm{~cm}$ como risco muito aumentado para DCV. (WHO, 1995).

Através do recordatório habitual de um dia, foram obtidos os relatos do consumo de colesterol, lipídios totais, ácidos graxos saturados, monoinsaturados e poli-insaturados. Posteriormente, por meio do software de nutrição DIETWIN ${ }^{\circledR}$ Profissional 1995/2002, obteve-se a quantificação desses nutrientes em gramas/ dia e porcentagem e, para o colesterol, em miligramas/dia. Esses valores foram comparados aos recomendados pela IV Diretriz Brasileira sobre Dislipidemia e Prevenção de Aterosclerose. Conforme essas recomendações, os valores preconizados são: lipídeos totais (25 a $35 \%$ das calorias totais), ácidos graxos saturados $(\leq 7 \%)$, ácidos graxos poli-insaturados ( $\leq$ $10 \%)$, ácidos graxos monoinsaturados ( $\leq$ $20 \%$ ) e colesterol ( $<200 \mathrm{mg} /$ dia). (MAIA; NICOLATO; LOPES, 2011; SPOSITO et al., 2007). 
A análise estatística dos dados foi realizada utilizando-se o programa IBM Statistical Package for the Social Science $\left(\right.$ SPSS $\left.^{\circledR}\right)$ versão 19.0. As variáveis quantitativas foram descritas através de média, mediana, mínimo, máximo e desvio padrão. As categorias foram descritas por meio de frequências absolutas e relativas. Para avaliar a associação entre as variáveis quantitativas, o coeficiente de correlação de Pearson foi aplicado. A associação entre as categóricas foi avaliada pelo teste qui-quadrado de Pearson. Para comparar médias entre os grupos, foi aplicado o teste ANOVA para amostras independentes. $\mathrm{O}$ nível de significância considerado foi de $5 \%$.

\section{Resultados}

Avaliou-se 90 idosos de ambos os gêneros, com idade média de $67,33 \pm 6,24$ anos (mínima $=60$ e máxima $=86$ anos). Ao categorizar as faixas etárias, verificou-se que $72,2 \%$ tinham $60-69$ anos. Em relação ao estado civil, 54,4\% eram casados e $34,4 \%$, viúvos. No que diz respeito à renda, $35,6 \%$ relatou ganhar entre 2 e 4 salários-mínimos (s.m.) e $46,7 \%$ mencionaram receber mais de 4 s.m. Quanto à escolaridade, 27,8\% possuem nível superior e $21,1 \%$, ensino fundamental incompleto.

As variáveis antropométricas mostram, por meio da CC, que $71,1 \%$ dos idosos classificaram-se em risco aumentado e muito aumentado para DCV, tendo sido a CC média de $88,48 \pm 11,82$ cm. Já o IMC médio foi de $28,28 \pm 5,14$ $\mathrm{kg} / \mathrm{m}^{2}$, tendo $35,6 \%$ apresentado eutrofia e $56,7 \%$, obesidade.

Quando se comparou o IMC com idade, escolaridade, renda e estado civil, observou-se que $34,4 \%$ dos idosos com idade entre 60 e 69 anos estavam com IMC classificado como obesidade e $32,2 \%$ como eutrofia. Para os idosos com $\geq 70$ anos, a maior ocorrência foi de obesidade, com $22,2 \%$. Em relação ao nível de escolaridade, $18,8 \%$ dos idosos com ensino médio eram obesos e $13,3 \%$ dos com ensino superior eram eutróficos. Considerando a renda, $25,5 \%$ dos idosos que recebiam mais que 4 s.m. eram obesos, enquanto, entre os que recebiam de 2 a 4 s.m., $20 \%$ eram obesos. No que se refere ao estado civil, $27,7 \%$ dos viúvos eram obesos (Tabela 1). 
Tabela 1. Comparação de IMC com idade, escolaridade, renda e estado civil.

\begin{tabular}{|c|c|c|c|c|}
\hline Variáveis & & IMC & & \\
\hline & Desnutrição & Eutrofia & Obesidade & $p^{*} 0,05$ \\
\hline Idade & $n(\%)$ & $\mathrm{n}(\%)$ & $\mathrm{n}(\%)$ & 0,008 \\
\hline $60-69$ anos & $5(5,5)$ & $29(32,2)$ & $31(34,4)$ & \\
\hline$\geq 70$ anos & $2(2,2)$ & $3(3,3)$ & $20(22,2)$ & \\
\hline Nível de escolaridade & & & & 0,058 \\
\hline Ensino fund. incompleto & $0(0,0)$ & $6(6,6)$ & $13(14,4)$ & \\
\hline Ensino fundamental & $0(0,0)$ & $6(6,6)$ & $12(13,3)$ & \\
\hline Ensino médio & $3(3,3)$ & $8(8,8)$ & $17(18,8)$ & \\
\hline Ensino superior & $4(4,4)$ & $12(13,3)$ & $9(10,0)$ & \\
\hline Renda & & & & 0,405 \\
\hline Até 1 s.m. & $0(0,0)$ & $2(2,2)$ & $4(4,4)$ & \\
\hline $1-2$ s.m. & $0(0,0)$ & $4(4,4)$ & $6(6,6)$ & \\
\hline 2-4 s.m. & $1(1,1)$ & $13(14,4)$ & $18(20,0)$ & \\
\hline$>4$ s.m. & $6(6,6)$ & $13(14,4)$ & $23(25,5)$ & \\
\hline Estado civil & & & & 0,003 \\
\hline Solteiro & $0(0,0)$ & $0(0,0)$ & $2(2,2)$ & \\
\hline Casado & $5(5,5)$ & $22(24,4)$ & $22(24,4)$ & \\
\hline Divorciado & $2(2,2)$ & $4(4,4)$ & $2(2,2)$ & \\
\hline Viúvo & $0(0,0)$ & $6(6,6)$ & $25(27,7)$ & \\
\hline
\end{tabular}

s.m= salário mínimo; fund.=fundamental; * teste qui-quadrado de Pearson.

Em relação ao consumo de lipídeos totais, a média foi de $19,63 \pm 10,74 \%$. Apenas 8,9\% dos idosos consumiram acima do recomendado, e $15,6 \%$, dentro do preconizado. Comparando-se o consumo de ácidos graxos monoinsaturados com o recomendado, verificou-se que $96,7 \%$ atendem às recomendações. Categorizando essa recomendação em partes, observou-se que $45,6 \%$ consomem de 0 a $5 \%$. Para os ácidos graxos poli-insaturados, $93,3 \%$ dos idosos atenderam às recomendações, tendo $72,2 \%$ consumido de 0 a $5 \%$, e a média foi de $7,3 \pm 7,2 \mathrm{~g} /$ dia. Quanto aos ácidos graxos saturados, observou-se consumo médio de $8,26 \pm 4,2 \%$ e $14,6 \pm 10,4 \mathrm{~g} /$ dia. Destes, $41,1 \%$ consumiam dentro do preconizado, mas $32,2 \%$ dos idosos consumiam acima. No que concerne ao consumo de colesterol, verificou-se que $72,2 \%$ consumiam dentro do recomendado (Tabela 2). 
Tabela 2. Características de consumo de lipídeos, ácidos graxos e colesterol dos idosos participantes do Projeto NUTENV da Universidade de Caxias do Sul.

\begin{tabular}{|c|c|c|c|c|}
\hline Variáveis & n (\%) & Média $\pm D P$ & Mín. Máx. & Mediana \\
\hline Gorduras totais $(\mathrm{g})$ & & $34,4 \pm 21,4$ & & \\
\hline Gorduras totais (\%) & & $19,63 \pm 10,74$ & $1,37-55,06$ & 18,26 \\
\hline $25 \%-35 \%$ & $14(15,6)$ & & & \\
\hline$<25 \%$ & $68(75,6)$ & & & \\
\hline$>35 \%$ & $8(8,9)$ & & & \\
\hline A.g. monoinsaturado (g) & & $12,4 \pm 9,1$ & & \\
\hline A.g. monoinsaturado (\%) & & $7,23 \pm 5,28$ & $0,24-28,04$ & 5,70 \\
\hline $0-5 \%$ & $41(45,6)$ & & & \\
\hline $5,1-10 \%$ & $29(32,2)$ & & & \\
\hline $10,1-20,0 \%$ & $17(18,9)$ & & & \\
\hline$>20 \%$ & $3(3,3)$ & & & \\
\hline A.g. poli-insaturado (g) & & $7,3 \pm 7,2$ & & \\
\hline A.g. poli-insaturado (\%) & & $4,14 \pm 4,16$ & $0,30-24,07$ & 2,52 \\
\hline $0-5 \%$ & $65(72,2)$ & & & \\
\hline $5,1-10$ & $19(21,1)$ & & & \\
\hline$>10 \%$ & $6(6,7)$ & & & \\
\hline A.g. saturado (g) & & $14,6 \pm 10,4$ & & \\
\hline A.g. saturado & & $8,26 \pm 4,21$ & $0,46-27,14$ & 7,30 \\
\hline Até $7 \%$ & $37(41,1)$ & & & \\
\hline $7,1-10 \%$ & $24(26,7)$ & & & \\
\hline$>10,1 \%$ & $29(32,2)$ & & & \\
\hline Colesterol & & $177,38 \pm 153,50$ & $0,44-820,53$ & 133,70 \\
\hline Preconizado ( $\leq 200$ mg) & $65(72,2)$ & & & \\
\hline Acima preconizado & $25(27,8)$ & & & \\
\hline
\end{tabular}

$\mathrm{n}=$ número; $\mathrm{DP}=$ desvio padrão; \%= porcentagem; A.g.= ácido graxo, g= gramas; $\mathrm{mg}=$ miligramas .

Com relação à associação do consumo de lipídeos, ácidos graxos saturados, monoinsaturados, poli-insaturados e colesterol com idade, escolaridade, renda, estado civil, observou-se que não houve diferença significativa entre os grupos.

Correlacionando o consumo de lipídeos com IMC e CC, idade com IMC e consumo de lipídeos, ácidos graxos saturados, monoinsaturados, poli-insaturados e colesterol, houve significância apenas para IMC e idade, onde ficou evidenciado que, quanto maior a idade, maior é o IMC. Houve significância negativa onde se observou que, quanto maior o consumo de lipídeos, menor o IMC, demonstrando, nesse resultado, um viés de causalidade reversa (Tabela 3 ). 
Tabela 3: Correlação do consumo de lipídeos com IMCe CC, idade com IMC e consumo de lipídeos, ácidos graxos e colesterol.

\begin{tabular}{lcc}
\hline Variáveis & Lipídeos/r $\left(\mathrm{p}^{*} 0,05\right)$ & Idade/r $\left(\mathrm{p}^{*} 0,05\right)$ \\
\hline IMC & $-0,256(0,015)$ & $0,208(0,049)$ \\
CC & $-0,185(0,081)$ & \\
Lipídeos & $-0,111(0,299)$ \\
A.g. & $-0,036(0,736)$ \\
monoinsaturado & $-0,064(0,549)$ \\
$\begin{array}{l}\text { A.g. } \\
\text { poli-insaturado }\end{array}$ & $-0,145(0,173)$ \\
$\begin{array}{l}\text { A.g. saturado } \\
\text { Colesterol }\end{array}$ & $-0,012(0,914)$ \\
\hline $\begin{array}{l}\text { r = símbolo do coeficiente de correlação Pearson; } \mathrm{p}= \\
<0,05 ; \text { IMC= índice de massa corporal, CC }=\text { circunferên- } \\
\text { cia da cintura, A.g.= ácido graxo. }\end{array}$
\end{tabular}

\section{Discussão}

No presente estudo, foram avaliados 90 idosos, de ambos os sexos, com maior prevalência de mulheres $(\mathrm{n}=82)$. O predomínio de idosos do sexo feminino $(91,1 \%)$ sugere que as mulheres são mais participativas das atividades socioculturais e estão mais sensibilizadas às necessidades demandadas para a promoção da saúde. Essa tendência mundial de maior participação das mulheres também foi apontada por Cervato et al. (2005), ao avaliarem uma intervenção educativa entre idosos frequentadores de Universidades Abertas para a Terceira Idade no município de São Paulo (SP), em que $93 \%$ das participantes eram do gênero feminino.

Os avaliados neste estudo, em sua maioria, eram casados e viúvos, o que coincide com a avaliação de Bueno et al. (2008), que, em estudo realizado em Alfenas(MG), com 85 idosos, encontrou $43,9 \%$ de casados e $35,4 \%$ de viúvos.
Ao avaliar a renda, encontrou-se que $46,7 \%$ dos idosos ganhavam valores superiores a 4 s.m. Estudo de Machado et al. (2009), com frequentadores do Centro de Doenças de Alzheimer e outras Doenças Mentais na Velhice da Universidade Federal do Rio de Janeiro, revelou que os idosos tinham renda superior a $5 \mathrm{~s}$. m. Já no estudo de Merle et al. (2011), realizado na França, com 964 idosos, a renda variou de 3,1 e 9,4 s.m. mensais. A literatura, como se percebe, aponta um perfil de idosos com condições financeiras de manter cuidados com a saúde e o mínimo para uma alimentação adequada, aproximando-se da realidade dos avaliados no presente estudo.

Os idosos que participam de UNTI, geralmente, possuem melhor nível de escolaridade. Nesta pesquisa, $27,8 \%$ dos avaliados tinham nível superior. Dado semelhante foi encontrado por Merle et al. (2011), em cujo estudo $32,5 \%$ possuíam nível universitário. De acordo com Rossato et al. (2010), esse dado foi prevalente nas cidades de São Leopoldo e Porto Alegre, onde 58,3\% estudaram mais de 12 anos.

Em relação à avaliação antropométrica, observou-se que os idosos, em sua maioria, eram obesos, fato compatível com os achados de Bueno et al. (2008), que encontraram prevalência de sobrepeso. Lowe et al. (2011), em estudo realizado no Reino Unido com 608 mulheres adultas e idosas, encontraram IMC médio de 27,30 $\pm 5,70$, sendo classificadas em sobrepeso e obesidade. Na Itália, a obesidade estava presente em $62,5 \%$ dos idosos avaliados. (CEREDA et al., 2011). 
Segundo Prentice et al. (2011), em estudo com 450 mulheres na pós-menopausa, em Washington, a prevalência de obesidade foi de $36,6 \%$. Esse dado também foi publicado por Gonçalves et al. (2012) em seu estudo transversal com 93 idosos, em que a maioria era obesa. Em Viçosa - MG, em um estudo realizado com diabéticos da terceira idade, 40,9\% dos idosos eram eutróficos e apenas 22,7\% eram obesos. (TEIXEIRA; SILVA; ALFENAS, 2009). Onisto et al. (2009), em estudo desenvolvido na Itália com 192 mulheres revelou um IMC médio de $28,4 \mathrm{~kg} / \mathrm{m}^{2}$ entre idosas diabéticas e $26,3 \mathrm{~kg} / \mathrm{m}^{2}$ entre não diabéticas. Esses indícios apontam que os valores de IMC para a maioria dos idosos aumentam com a idade, ao passo que a estatura e a quantidade de massa magra diminuem. Em vários estudos, pode-se observar prevalência da obesidade e sobrepeso. Esse é um dado relevante, considerando que se trata de um fator de risco importante para DCV e doenças crônicas não transmissíveis. (PIATI; FELICETTI; LOPES, 2009; BORTOLI et al., 2011).

Em relação à avaliação da $\mathrm{CC}$, observou-se em $71,1 \%$ dos idosos valores relacionados a risco elevado ou muito elevado para DCV. Bortoli et al. (2011) encontraram, em sua amostra, 60,2\% de indivíduos apresentando risco muito aumentado para DCV, na cidade de Veranópolis. Estudo realizado em Antônio Prado - RS, com 22 idosas, obteve valores médios de 95,2 cm de CC, apresentando risco muito elevado. (FARINEA; RICALDI; SIVIERO, 2010). Conforme Tyrovolas et al. (2011), em pesquisa realizada na Grécia, os 1.190 participantes apresen- tavam valores variando de 104 a 103 $\mathrm{cm}$, também com risco muito elevado. Outra publicação realizada em Cuba, com total de 1.905 participantes, foram verificadas CC entre 87,1-92,2 $\mathrm{cm}$ para homens e 83,96-90,32 cm para mulheres. (COQUEIRO; BARBOSA; BORGATTO, 2009).

No presente estudo, os resultados de obesidade e CC elevada vêm ao encontro do aumento da gordura, sobretudo abdominal, o que é preocupante na maioria dos idosos, pois implica risco de DCV. Obteve-se, ainda, correlação positiva entre CC e consumo de lipídeos, que também pode estar relacionado a essas doenças.

Quanto aos lipídeos totais, este estudo observou que 75,6\% dos participantes os consumiam abaixo do recomendado, diferentemente do que verificaram Souza et al. (2010), em estudo realizado no Rio de Janeiro, em que o consumo ficou dentro do preconizado, sendo $28,1 \%$ para o sexo feminino e $25,9 \%$ para o masculino. O consumo de lipídeos no presente estudo foi de 34,4 g/dia, resultados abaixo dos valores encontrados por Larsson, Virtamo e Wolk, (2012), em trabalho realizado na Suécia, onde constataram consumo de $45,7 \mathrm{~g} /$ dia. Wyka et al. (2012), em publicação na Polônia, com 238 idosos, encontrou consumo de 60,3 e $50,3 \mathrm{~g} /$ dia para homens e mulheres, respectivamente. Para Ruano et al. (2011), em estudo na Espanha, os valores de consumo chegaram a $113,7 \mathrm{~g} /$ dia.

Segundo nossos achados, $41,1 \%$ consumiram menos de $7 \%$ de ácidos graxos saturados, ficando dentro do recomendado, enquanto $58,9 \%$ os consumiam acima desse parâmetro. Bortoli et al. 
(2011), avaliando 116 indivíduos idosos, observaram que a maioria consumia ácidos graxos saturados de acordo com o preconizado. Ao contrário, o estudo de Naqvi et al. (2012), com 482 mulheres, obteve consumo médio de $11,1 \%$. O consumo médio de ácidos graxos saturados no presente estudo foi de $14,7 \mathrm{~g}$, abaixo do encontrado por outros estudos: Gopinath et al. (2011) encontraram valores variando de $20,8 \mathrm{~g}$ a $36,3 \mathrm{~g}$, enquanto Ruano et al. (2011) verificaram médias de consumo entre 22,8 e $45,5 \mathrm{~g} /$ dia.

Quanto ao consumo de ácidos graxos monoinsaturados, $96,7 \%$ dos idosos avaliados neste estudo os consumiam dentro dos valores recomendados. Naqvi et al. (2012), em estudo realizado em Boston com 1.201 mulheres, obtiveram consumo médio de $11,5 \%$ desses ácidos graxos na dieta. Considerando o consumo em gramas, no presente estudo, foi obtido um valor de 12,5 g/dia. Gopinath et al. (2011) encontraram consumo mediano variando de $19,8 \mathrm{~g}$ a $32,9 \mathrm{~g}$ por dia. Larsson, Vertigo e Wonk (2012) encontraram o consumo médio de $15,4 \mathrm{~g}$.

Neste estudo, o consumo de ácidos graxos poli-insaturados para $93,3 \%$ dos avaliados ficou dentro do recomendado, sendo a média de 7,3 g/dia, resultado próximo ao verificado por Larsson, Vertigo e Wonk (2012), com 7,1 g/dia. Tan et al. (2012) encontraram mediana de consumo de 5,3 g/dia em estudo realizado em Los Angeles com 1.575 participantes. Ruano et al. (2011), por sua vez, constataram 9,0 e 20,5 g/dia. Os ácidos graxos poli-insaturados estão presentes em diversos alimentos que compõem o padrão alimentar dos indivíduos e estão associados à redução do risco para DCV.

$O$ colesterol tem função estrutural, sendo constituinte de membranas celulares e camadas que revestem as lipoproteínas. Trata-se, também, de um precursor de ácidos biliares, hormônios esteroides e vitamina D. As lipoproteínas plasmáticas apresentam diferentes densidades, de acordo com a quantidade de proteínas que as compõem. (SILVA, 2005). Neste estudo, o consumo alimentar de colesterol foi de $177,3 \mathrm{mg}$, tendo $72,2 \%$ da amostra consumido dentro do preconizado. Esses resultados são próximos aos divulgados por Larsson, Vertigo e Wonk (2012), com consumo médio de $187 \mathrm{mg}$. Valores similares também foram obtidos por Tan et al. (2012), com 186 mg/dia. Nagtsuyu et al. (2009), analisando 98 idosos em Ribeirão Preto, encontraram consumo médio de 240,95 $\mathrm{mg} /$ dia, o que está acima do desejado. Estudo desenvolvido na Austrália observou mediana de 241-339 mg/dia de consumo de colesterol total. (GOPINATH et al., 2011). Lim et al. (2012), em pesquisa realizada na Coreia do Sul com 607 idosos, encontraram média de 190,7 mg para homens e $211,9 \mathrm{mg}$ para mulheres, tendo estas consumido colesterol acima do recomendado.

Em nosso estudo, foi utilizado para avaliação da ingestão de alimentos 0 inquérito alimentar recordatório de 24 horas, onde o entrevistado relatava a ingestão alimentar habitual de um dia. Por ser de fácil aplicação e estar baseado no relato dos participantes, esse método foi empregado em várias publicações. (PRENTICE et al., 2011; LOWE et al., 
2011; MERLE et al., 2011; ROSSATO et al., 2010; SOUZA et al., 2010).

Dentre as limitações deste trabalho, pode-se destacar que, por se tratar de um estudo transversal, há um aumento da chance de ocorrer um viés de causalidade reversa. Outra limitação corresponde ao fato de a avaliação alimentar ter sido realizada por meio de um recordatório de 24 horas de apenas um dia e de os nutrientes terem sido calculados com auxílio de um programa informatizado, no qual várias tabelas de composição centesimal dos alimentos são utilizadas. A dificuldade de expressar o tamanho da porção caseira do alimento consumido, que poderia ser limitante à maioria dos idosos, não foi considerada, uma vez que os avaliados possuíam bom nível de escolaridade e não apresentavam deficit cognitivo.

\section{Conclusão}

A amostra avaliada foi constituída por idosos de ambos os sexos, participantes do Projeto NUTENV. Em sua maioria, eram mulheres, casados, com renda mensal de 2 a 4 salários mínimos e mais de 8 anos de escolaridade. Houve prevalência de sobrepeso e obesidade, assim como maiores valores de CC.

Em relação aos lipídeos, observou-se que os ácidos graxos monoinsaturados, poli-insaturados e saturados, assim como o colesterol, ficaram dentro do preconizado, porém os lipídeos totais, obtidos em porcentagem, ficaram abaixo do esperado. Quando correlacionado esse consumo com o IMC, houve significância negativa, registrando-se que, quanto maior o consumo de lipídeos, menor o IMC.

Com relação aos dados obtidos, sugere-se a adoção de hábitos alimentares saudáveis, adequados em macro e micronutrientes, associada à prática de atividade física para diminuição da adiposidade corporal, principalmente por meio da circunferência da cintura. Além disso, apesar de a literatura apontar que, com o processo de envelhecimento, ocorrem alterações biológicas corporais, como o ganho de massa adiposa, esse fato é preocupante, pois se trata de um fator de risco para as doenças crônicas, como foi encontrado neste estudo através das medidas do IMC e CC.

Recomenda-se que novos estudos sejam realizados ampliando a amostra e relacionando como a nutrição pode prevenir as doenças cardiovasculares. Ressalta-se, ainda, que modificações nos hábitos dietéticos, especialmente no consumo de lipídeos, ácidos graxos saturados e de colesterol, podem prevenir doenças coronarianas. O controle do consumo desses nutrientes pode diminuir os riscos de complicação relacionada às doenças cardiovasculares. Nesse sentido, para os avaliados nesta amostra, os resultados podem representar um fator de proteção, já que a maioria os consome dentro do esperado. 


\section{Consumption of lipids and nutritional status of elderly project participants NUTENV University of Caxias do Sul}

\section{Abstract}

The aim of this study was to assess the dietary intake of fat, monounsaturated fatty acids, polyunsaturated, saturated and cholesterol and nutritional status by anthropometry senior project participants NUTENV the University of Caxias do Sul / RS. It is a cross-sectional, retrospective, descriptive and analytical, with 90 elderly of both sexes. We analyzed demographic, socioeconomic and food consumption. Regarding anthropometric evaluated $\mathrm{BMI}$ and WC. To estimate the dietary used the R24 hr. Statistical analysis involved the chi-square test for association of the anthropometric (BMI and WC) with demographic and socioeconomic variables and the Pearson correlation coefficient to correlate lipid intake with anthropometric and demographic variable age. The consumption of lipids was described as mean, median, minimum, maximum and standard deviation. Among the subjects studied were of females, married, with monthly income between 2 and $04 \mathrm{sm}$, and more than 8 years of schooling. Anthropometric evaluation $71.1 \%$ of individuals are at high risk for CVD or very high, considering the CC. BMI obtained by a higher frequency of obesity $(56.7 \%)$. The consumption of total lipids was lower than recommended for most individuals analyzed. For fatty acids and cholesterol obtained results within the recommended intake. The association of variables, the results showed a higher prevalence of obesity among elderly with 60-69 and widowers. There was significant negative correlation between lipid intake and BMI, and higher lipid intake lower BMI. Becomes necessary to adopt healthy eating habits with a balanced diet of macro and micronutrients associated with physical activity practice, as one of the goals of decreasing WC and BMI. It is also suggested that further studies are linking the consumption of lipids in individuals in the aging process, using other assessment tools.

Keywords: Fatty acids. Aging. Nutritional status. Elderly.

\section{Referências}

BORTOLI, C. et al. Ingestão dietética de gordura saturada e carboidratos em adultos e idosos com dislipidemia oriundos do Projeto Veranópolis. Revista Brasileira de Cardiologia, Rio de Janeiro, v. 24, n. 1, p. 33-41, jan./fev. 2011.

BUENO, J. M. et al. Avaliação nutricional e prevalência de doenças crônicas não transmissíveis em idosos pertencentes a um programa assistencial. Ciência \& Saúde Coleti$v a$, Rio de Janeiro, v. 13, n. 4, p. 1237-1246, 2008.

CEREDA, E. et al. Nutritional screening and mortality in newly institutionalised elderly: a comparison between the geriatric nutritional risk index and the mini nutritional assessment. Clinical Nutrition, Philadelphia, v. 30, Issue 6, p. 793-798, 2011.

CERVATO, A. M. et al. Educação nutricional para adultos e idosos: uma experiência positiva em Universidade Aberta para a Terceira Idade. Revista de Nutrição, Campinas, v. 18, n. 1, p. 41-52, jan./fev. 2005.

COQUEIRO, R. S.; BARBOSA, A. R.; BORGATTO, A. F. Anthropometric measurements in the elderly of Havana, Cuba: age and sex differences. Nutrition, v. 25, n. 1, p. 33-39, 2009 .

DURO, L. N.; ASSUNÇÃO, M. C.; COSTA, J. S. D.; SANTOS, I. S. Desempenho da solicitação do perfil lipídico entre os setores público e privado. Rev Saúde Pública, v. 42, n. 1, p. 82-88, 2008. 
FARINEA, N.; RICALDE, S. R.; SIVIERO, J. Perfil nutricional e antropométrico de idosos participantes de um grupo de ginástica no município de Antônio Prado - RS. Revista Brasileira de Ciências do Envelhecimento Humano, Passo Fundo, v. 7, n. 3, p. 394-405, set./dez. 2010.

GONÇALVES, D. F. et al. Nutricional status and epidemiological profile of elderly people. Archives of Gerontology and Geriatrics, Philadelphia, v. 55, p. 1-4, 2012.

GOPINATH, B. et al. Dietary intake of cholesterol is positively associated and use of cholesterol-lowering medication is negatively associated with prevalent age-related hearing loss. The Journal of Nutrition - Nutritional Epidemiology, v. 141, n. 7, p. 1355-1361, 2011.

JENKINS, D. J. A. et al. Adding monounsaturated fatty acids to a dietary portfolio of cholesterol-lowering foods in hypercholesterolemia. Canadian Medical Association or its licensors, Ottawa, v. 182, n. 18, p. 1961-1967, dec. 2010.

KARVETTI, R. L.; KNUTS, L. R. Validity of the 24-hour dietary recall. J Am Diet Assoc, v. 85, n. 11 , p. 1437-1442, 1985.

LARSSON, S. C.; VIRTAMO, J.; WOLK, A. Dietary fats and dietary cholesterol and risk of stroke in women. Atherosclerosis, Philadelphia, v. 221, Issue 1, p. 282-286, 2012.

LATREILLE, J. et al. Dietary monounsaturated fatty acids intake and risk of skin photoaging. Journal PLoS ONE, v. 7, Essue 9, p. 1-7, sep. 2012.

LIM, S. et al. Optimal cut points of waist circumference(WC) and visceral fat area (VFA) predicting for metabolic syndrome (MetS) in elderly population in the Korean longitudinal Study on health and Aging (KLoSHA). Archives of Gerontology and Geriatrics, v. 54, p. e29-e34, 2012.

LIPSCHITZ, D. A. Screening for nutritional status in the elderly. Primary Care, v. 21, n. 1, p. 55-67, 1994.
LOWE, N. M. et al. Dietary calcium intake, vitamin d status, and bone health in postmenopausal women in rural Pakistan. Journal Population Nutrition, v. 29, n. 5, p. 465-470, oct. 2011.

MACHADO, J. et al. Estado nutricional na Doença de Alzheimer. Revista Associação Médica Brasileira, São Paulo, v. 55, n. 2, p. 188-191, 2009.

MACHADO, J. S.; FRANK, A. A.; SOARES, E. A. Fatores dietéticos relacionados à Doença de Alzheimer. Revista Brasileira de Nutrição Clínica, São Paulo, v. 21, n. 3, p. 252-257, 2006.

MAIA, I. C. M. P.; NICOLATO, R.; LOPES, A. C. S. Aconselhamento nutricional a idosos dislipidêmicos. Revista Médica de Minas Gerais, Minas Gerais, v. 21, n. 3, p. 253-259, 2011.

MERLE, B. et al. Dietary omega-3 fatty acids and the risk for age-related maculopathy: the alienor study. Clinical and Epidemiologic Research, v. 52, n. 8, p. 6004-6010, jul. 2011.

NAGATSUYU, D. T. et al. O impacto da obesidade abdominal sobre os níveis plasmáticos de lipídeos nos idosos. Revista da Universidade de Medicina de Ribeirão Preto, Ribeirão Preto, v. 42, n. 2, p. 157-163, 2009.

NAQVI, A. Z. et al. Monounsaturated, trans $\&$ saturated fatty acids and cognitive decline in women. Journal American Geriatric, v. 59, n. 5, p. 837-843, May 2012.

ONISTO, N. et al. Anthropometric traits and aging: a cross-sectional survey in diabetic elderly women. Archives of Gerontology and Geriatrics, v. 48, Issue 2, p. 197-200, 2009.

PIATI, J.; FELICETTI, C. R.; LOPES, A. C. Perfil nutricional de hipertensos acompanhados pelo Hiperdia em Unidade Básica de Saúde de cidade paranaense. Revista Brasileira de Hipertensão, Rio de Janeiro, v. 16, n. 2 , p. 123-129, 2009. 
PRENTICE, R. L. et al. Evaluation and comparison of food records, recalls, and frequencies for energy and protein assessment by using recovery biomarkers. American Journal of Epidemiology, v. 174, n. 5, p. 591-603, 2011.

ROQUE, F. P.; BOMFIM, F. M. S.; CHIARI, B. M. Descrição da dinâmica de alimentação de idosas institucionalizadas. Revista Sociedade Brasileira de Fonoaudiologia, São Paulo, v. 15, n. 2, p. 256-263, 2010.

ROSSATO, S. L. et al. Seasonal effect on nutrient intake in adults living in Sothern Brazil. Caderno de Saúde Pública, Rio de Janeiro, v. 26, n. 11, p. 2177-2187, nov. 2010.

RUANO, C. et al. Dietary fat intake and quality of life: the SUN project. Nutrition Journal, v. 10, n. 121, p. 1-8, 2011.

SILVA, E. B. Estudo do perfil lipídico de um grupo de idosos. Revista Newslab, São Paulo, Edição 72, p. 142-158, 2005.

SILVA, I. M. C.; SÁ, E. Q. C. Alimentos funcionais: um enfoque gerontológico. Revista Brasileira de Clínica Médica, São Paulo, v. 10, n. 1, p. 24-28, jan./fev. 2012.

SOUZA, D. R. et al. Ingestão alimentar e balanço energético da população adulta de Niterói, Rio de Janeiro, Brasil: resultados da Pesquisa de Nutrição, Atividade Física e Saúde (PNAFS). Caderno de Saúde Pública, Rio de Janeiro, v. 26, n. 5, p. 879-890, maio 2010.

SPOSITO, A. C. et al. IV Diretriz Brasileira sobre Dislipidemias e Prevenção da Aterosclerose. Departamento de Aterosclerose da Sociedade Brasileira de Cardiologia. Arquivos Brasileiros de Cardiologia, Rio de Janeiro, v. 88, Supl. I, p. 2-19, abr. 2007.

TAN, Z. S. et al. Red blood cell omega-3 fatty acid levels and markers of accelerated brain aging. American Academy of Neurology, v. 78, n. 9, p. 658-664, Feb. 2012.
TEIXEIRA, K. R.; SILVA, M. F.; ALFENAS, R. C. G. Educação nutricional continuada no controle do diabetes em idosos. Revista Participação da UNB, Brasília, ano 9, n. 15, p. 76-83, jul. 2009.

TYROVOLAS, S. et al. Nutrition intake in relation to central and overall obesity status among elderly people living in the Mediterranean islands: the MEDIS study. Nutrition, Metabolism \& Cardiovascular Diseases, v. 21, Issue 6, p. 438-445, 2011.

WORLD HEALTH ORGANIZATION. Physical status: the use and interpretation of anthropometry. WHO Technical Report Series 854. Geneva: WHO, 1995.

WYKA, J. et al. Assessment of dietary intake and nutritional status (MNA) in Polish free-living elderly people from rural environments. Archives of Gerontology and Geriatrics, v. 54, Issue 1, p. 44-49, 2012. 